\title{
¡Todos(as) emprendedores(as)! Subjetividad y salud mental en un proceso de capacitación en emprendimiento*
}

\section{All entrepreneurs! Subjectivity and mental health in a process of entrepreneurship training}

\author{
Ximena Castro-Sardi ${ }^{1}$ \\ Universidad Icesi, Cali, Colombia \\ xcastro@icesi.edu.co \\ https://orcid.org/0000-0002-0740-9293
}

Yuli Andrea Salazar ${ }^{2}$

Universidad Icesi, Cali, Colombia

yuli.salazar.valencia@gmail.com

https://orcid.org/ 0000-0002-9899-348X

\section{Margarita M. Munévar ${ }^{3}$}

Universidad Icesi, Cali, Colombia

mmmunevar@icesi.edu.co

https://orcid.org/0000-0003-0418-3070

Recibido: 31-07-19

Aprobado: 10-06-20

\footnotetext{
* Esta investigación fue financiada por la Universidad Icesi (Cali, Colombia), en el marco de su convocatoria interna del año 2017. La investigación se llevó a cabo entre septiembre de 2017 y agosto de 2018.

1 Máster en Ciencias Sociales y en Psicoanálisis.

2 Especialista en Cooperación y Gerencia Social.

3 Máster en Intervención Psicosocial.
} 


\section{Resumen}

Este artículo presenta los resultados de un proceso de investigación-intervención cuyo objetivo fue ofrecer atención psicosocial a un grupo de personas, en su mayoría mujeres, vinculadas a un programa de formación para el emprendimiento en Cali. Se identifican las principales problemáticas de salud mental y los efectos del proceso de atención, mediante entrevistas en profundidad y un cuestionario de bienestar psicológico a una muestra de participantes. Las problemáticas identificadas son la sobrecarga por parte de las mujeres en su rol familiar y negocio, la dificultad para delegar y tomar decisiones y la vivencia de situaciones de maltrato familiar. Los principales resultados de la intervención son la movilización hacia la toma de decisiones, la delegación de funciones, el desarrollo de auto-confianza y la búsqueda activa de soluciones a situaciones de maltrato. Se concluye sobre la necesidad de incluir aspectos de la experiencia subjetiva en los programas de intervención basados en el emprendimiento.

Palabras clave: empresarios; capacitación; programa social; salud mental; bienestar social.

\section{Abstract}

This paper presents the results of a research-intervention process which objective was to offer psycho-social attention to a group of people, the majority of which were women, linked to a formation program for entrepreneurship in Cali. The main issues of mental health and the effects of attention process are identified, through interviews in depth and a survey of a psychological well-being to a sample of the participants. The identified problems are the overload of women in their family and business role, the difficulty to delegate and make decisions, and the experiencing of family abuse predicaments. The main results of the intervention are the mobilization towards decision making, the delegation of functions, the development of self-confidence and the active search of solutions regarding situations of abuse. It concludes on the need to include aspects of subjective experience in entrepreneurship-based intervention programs.

Keywords: entrepreneurs; training; social program; mental health; social well-being. 


\section{Introducción}

El presente artículo es resultado de una investigación-intervención realizada durante el año 2018, en Cali, a partir de un proceso de atención psicosocial a un grupo de 95 personas. En su mayoría, mujeres inscritas en un programa de capacitación para el emprendimiento (PCE) de una institución privada de la ciudad ${ }^{4}$. Concernidas por la alta deserción en sus programas de capacitación, y preocupadas por problemáticas familiares y de salud mental de sus beneficiarias, las directivas de esta institución establecieron una alianza con el Consultorio de Atención Psicosocial (CAPsi) de la Universidad Icesi, de Cali. Con el fin de proveer un servicio profesional de escucha y orientación psicológica a las beneficiarias de sus programas que lo solicitaran. Paralelamente a la intervención, se realizó una sistematización de la experiencia con fines investigativos. Las preguntas que orientaron inicialmente esta sistematización fueron: ¿Qué problemáticas psicosociales inciden y de qué manera en los procesos de emprendimiento? ¿Qué relación existe entre el empoderamiento en las mujeres y sus proyectos de emprendimiento? ¿Cómo se relacionan las problemáticas de salud mental con las trayectorias de emprendimiento?

Sobre el contexto del emprendimiento en Colombia, Arbeláez (2019) señala que solamente el $6 \%$ de las empresas creadas continúan funcionando más allá de los 3,5 años. Lo que puede estar relacionado con que gran parte la actividad emprendedora se realiza por necesidad ${ }^{5}$, tipo de emprendimiento que, en el $80 \%$ de los casos, conduce a la quiebra. Lo anterior profundiza las condiciones de pobreza de la población, teniendo en cuenta que esta actividad implica un alto grado de inversión financiera, personal y social.

4 En este artículo utilizaremos la sigla PCE para referirnos al programa específico de capacitación para el emprendimiento con el que se hizo la investigación-intervención.

5 Cuando se ejecuta una idea de negocio de forma, pero sin el total conocimiento de su potencial en el mercado, se puede decir que este tipo de emprendimiento es una aventura empresarial que se lleva a cabo por aquellos que buscan solucionar algún tipo de dificultad financiera o salir del desempleo.
Este panorama también se refleja igualmente en los niveles de deserción de los PCE, por lo que es menester contribuir a la comprensión de las causas de esta deserción, pero también de estrategias para mitigarla y promover el desarrollo exitoso de procesos de empoderamiento femenino a través del emprendimiento.

Al realizar una revisión de la producción investigativa sobre el tema, se encontró que la literatura dominante se enfoca principalmente en el análisis socio-económico del proceso de emprendimiento, la forma como se desarrolla el proceso en sus diferentes fases y cómo interactúan los diferentes componentes o fuerzas de la actividad empresarial durante estas fases (atributos individuales, valores sociales y condiciones del contexto empresarial).

Teniendo en cuenta los enfoques de investigación anteriores, este artículo se centrará en el componente de la actividad emprendedora denominado "atributos individuales". Al respecto, existen vastos estudios enfocados en la caracterización de los atributos psicológicos de la persona emprendedora. De los cuales los más explorados son: motivación al logro, locus de control y propensión al riesgo (Marulanda y Morales, 2016, p. 14).

El Global Entrepreneurship Monitor (GEM) -observatorio internacional en el tema de emprendimiento-, además de centrar algunos de sus análisis en los atributos psicológicos, tiene algunos informes, de publicación periódica, dedicados al tema de género desde el 2005. En el informe GEM (GERA, 2018), se destacan cuatro elementos relacionados con aspectos psicosociales del sujeto emprendedor y la perspectiva de género:

- Con un $22 \%$ de ocurrencia, la segunda causa para discontinuar una empresa, después de la falta de rentabilidad, son los motivos personales.

- La propuesta para abordar la "disparidad de género" en la actividad emprendedora se centra en el fortalecimiento de asertividad, confianza en sí mismas, percepción y manejo del riesgo, visión de carrera empresarial 
y conexiones empresariales. Esta propuesta concuerda con los resultados de la investigación realizada por Álvarez et al. (2012) en mujeres españolas, en donde se concluye que los "factores informales", tales como: redes sociales (conocimiento de alguien que tiene trayectoria emprendedora), autopercepción de habilidades emprendedoras (el individuo considera que tiene el conocimiento y habilidades para colocar en marcha un negocio) y rol familiar (nivel de dedicación a las labores del hogar), tienen efectos positivos/negativos significativos en la probabilidad de ser mujer emprendedora.

- En general, las propuestas para mitigar las dificultades personales de los emprendedores, para avanzar en el proceso empresarial, se centran en procesos educativos y de consejería.

Respecto a la relación entre emprendimiento, atención psicológica, bienestar psicológico y salud mental, el corpus investigativo dominante, según Stephan (2018), se ha enfocado en la descripción y caracterización, por medio de estudios transversales cuantitativos o mixtos, del bienestar mental de los emprendedores. Una de las críticas que se realiza a estos estudios es que se utilizan modelos e instrumentos de medición pensados para la empleabilidad; gran parte de estos estudios presentan análisis comparativos entre emprendedores y empleados.

De acuerdo a la revisión del estado del arte realizada por Stephan (2018), la mayor parte de los estudios se centran en las consecuencias positivas de un alto nivel de bienestar psicológico. Entre los temas poco estudiados, y que requieren más atención, se encuentran los siguientes: las consecuencias de la actividad emprendedora sobre el bienestar psicológico del emprendedor; la incidencia de trastornos mentales en esta población; las consecuencias del malestar psicológico en la actividad emprendedora; las consecuencias de un inadecuado equilibrio en las características psicológicas resaltadas como positivas (i.e. ¿Qué ocurre cuando no hay límites a la autonomía del emprendedor?). Se requieren entonces más estudios longitudinales $y$ cualitativos que describan el bienestar psicológico del emprendedor, sus necesidades y recursos, así como la variabilidad en los mismos.

Respecto a la literatura que relaciona específicamente emprendimiento y salud mental (Louie, 2015; Fowle, 2019; Dahl et al., 2010), la mayor parte se enfoca en "el rol de la salud mental y factores estresantes psicológicos en los emprendedores y sus efectos en el bienestar personal y el proceso de formación de la empresa" (Louie, 2015, p. 387). Sobre los posibles efectos negativos de los factores estresantes de la actividad emprendedora en la salud mental de las personas, algunos autores señalan: hay incremento en el consumo de medicamentos sedativos/hipnóticos, tanto en los emprendedores como en sus parejas sentimentales, lo que podría relacionarse con el incremento en los niveles de estrés (Dahl et al., 2010); en los emprendedores se aprecia una frecuencia más alta de problemas de salud mental en comparación con empleados (Gunnarsson et al., 2007); respecto a lo que Stephan (2018) ha llamado "funcionalidad" de problemas mentales en la actividad emprendedora, señala que los emprendedores tienen un alto nivel de incidencia de dislexia en comparación con empleados corporativos. Sin embargo, han desarrollado habilidades de afrontamiento que incluso llegan a ser benéficas para su negocio. Clark et al. (2008) encontraron que los emprendedores muestran más comportamiento de riesgo, más impulsividad personal y mayor flexibilidad cognitiva. Al respecto, los autores proponen la teoría de "impulsividad funcional", en la cual las características anteriores se convierten en fortalezas para el desarrollo del emprendimiento.

En general, desde la revisión de antecedentes, es importante resaltar que en la producción de conocimiento sobre la relación entre emprendimiento, salud mental o bienestar psicológico y género, existen tres aspectos poco estudiados: ¿cuáles son los efectos paradójicos de la actividad emprendedora sobre la salud mental o bienestar psicológico del sujeto?; ¿cómo interactúan las características psicológicas del sujeto emprendedor con las circunstancias de la actividad emprendedora?; y, desde una perspectiva de género, ¿cuáles son los efectos de la 
actividad emprendedora en mujeres con poco nivel de desarrollo de los denominados "factores informales"?

El proceso de investigación-intervención realizado aporta algunas respuestas a estas preguntas. Partimos del supuesto de que el desarrollo de proyectos de emprendimiento, así como el empoderamiento femenino que de estos puede derivarse, se relaciona con los recursos psíquicos, familiares y comunitarios de cada persona emprendedora. La sistematización del proceso de atención psicosocial, en el marco de este proyecto, contribuye a ampliar el conocimiento sobre las problemáticas en salud mental que enfrenta un grupo de personas en procesos de capacitación para el emprendimiento, particularmente mujeres; así como los efectos que tiene un dispositivo de escucha en la resolución de conflictos psíquicos y sociales que enfrentan las personas en sus trayectorias de emprendimiento.

\section{Marco teórico}

El proceso de investigación, asociado a la estrategia de atención en salud mental, se apoyó en dos ejes teóricos: el emprendimiento y sus componentes, la perspectiva de género, y la subjetividad en relación a la salud mental.

\subsection{Emprendimiento y género}

Según el GEM, el emprendimiento es "cualquier intento de creación de un nuevo negocio o una nueva empresa, tales como el autoempleo, una nueva organización comercial, o la expansión de un negocio ya existente, por un individuo, un equipo, o un negocio ya establecido" (GERA, s.f.).

El emprendimiento ha sido considerado como un mecanismo privilegiado para promover los procesos de empoderamiento de poblaciones vulnerables, especialmente mujeres. Además, es uno de los temas más estudiados en procesos de empoderamiento femenino (Narayan, 2005). Al respecto, es importante resaltar que, en estos procesos, tiende a asumirse que aquellas mujeres que son capaces de ganar o incrementar sus ingresos por sí mismas tienen la capacidad de situarse en una situación de poder; aunque en la práctica real de sus vidas es probable que esta relación no sea tan directamente proporcional, sino que dependa de otros factores como la cultura y la clase social, entre otros (Narayan, 2005; Rowlands, 1997).

El GEM plantea que el proceso de emprendimiento tiene cuatro componentes que se relacionan entre sí:

- Contexto social, cultural, político y económico.

- Valores sociales hacia el emprendimiento.

- Atributos individuales o factores psicológicos y motivacionales de los sujetos emprendedores.

- Actividad emprendedora, es el resultado de la interacción de los tres factores anteriores y se define a partir del ciclo de vida del emprendimiento, el tipo de actividad y el sector de la actividad.

Este marco propone los denominados "factores informales" como aquellos aspectos que afectan la actividad emprendedora, pero que no están tácitamente determinados por políticas institucionales formales. Algunos ejemplos de estos factores son: la percepción de habilidades, los roles familiares y las redes sociales (Álvarez et al., 2012). También son definidos como "el código moral del sistema de creencias" de una sociedad; algunos pueden ser transculturales, como específicos a cada sociedad (North, 2005, p. 50). Desde esta perspectiva, el emprendimiento se encuentra determinado tanto por los factores formales -políticas macro y micro económicas e índices de crecimiento económico- como por los factores informales, que hacen referencia a las relaciones familiares, de género y las expectativas sobre el rol de la mujer, entre otros.

El emprendimiento, sobre todo en población femenina, ha sido instrumentalizado como una vía privilegiada para promover procesos de desarrollo más eficientes y se tiende a asumir que generar procesos de desarrollo económico implica 
el empoderamiento de esta población. Esta tendencia surge entre 1970 y 1980 , cuando comienza la preocupación por los temas de género y su relación con el desarrollo. En este estudio, entendemos "género" como la forma en que se desarrollan las relaciones de poder entre hombres y mujeres, no solo a nivel de las relaciones personales, sino a nivel estructural en la sociedad (Rowlands, 1997).

La mayor parte de las investigaciones realizadas sobre la incidencia de los factores informales en el emprendimiento son de carácter cuantitativo, multifactorial, basadas en encuestas, con muestras estadísticamente significativas (Carter, 2000; Sorenson et al., 2008). Sin embargo, estas no abarcan con mayor profundidad los elementos más subjetivos, es decir, lo que significa para una persona emprender en relación con su historia de vida, y sus relaciones familiares y sociales.

\subsection{Subjetividad, salud mental y emprendimiento}

En la actualidad, las políticas públicas de desarrollo económico y social, mediante la generación de empleo y la promoción del emprendimiento, dan gran importancia a la calidad de vida, así como al bienestar psicosocial de las personas (Stephan, 2018). En Colombia, la Resolución 2646 de 2008 introduce los lineamientos para la identificación de los factores de riesgo psicosocial en el trabajo, y la Ley de salud mental -Ley 1616 de 2013- (Congreso de Colombia, 2013) señala explícitamente que las organizaciones deben velar por la salud mental y el bienestar psicosocial de sus empleados. El reconocimiento de los factores psicológicos en los procesos de empleabilidad y emprendimiento deriva entonces en la necesidad de definir una aproximación conceptual frente a estos, teniendo en cuenta que hay múltiples enfoques sobre la cuestión.

En este proceso de intervención-investigación adoptamos una postura basada en la salud mental comunitaria y el psicoanálisis aplicado, que propone una mirada sobre la subjetividad en la época actual, y su relación con la salud mental y el emprendimiento. Una perspectiva social-comunitaria de la salud mental implica:
[...] actuar en los sectores del tejido social donde se producen las fracturas de la relación humana, para ayudar a recuperar lo que entendemos como condición necesaria de un desarrollo subjetivo más pleno: el lazo social, la solidaridad grupal, la asunción colectiva de los problemas (Galende, 1997, p. 211).

En las sociedades contemporáneas, se evidencia "cierta reducción del sujeto a una especie de hombre-máquina, de quien se espera y exige eficacia, felicidad, y ausencia de conflictos y flaquezas" (Castro-Sardi, 2014, p. 119). Esta visión del sujeto está relacionada con una alianza entre positivismo científico y el capitalismo liberal, como orden socio-económico imperante (Galende, 2008; 2015). En este contexto, los abordajes sobre la salud mental de los sujetos en la actualidad tienden a dejar de lado la dimensión subjetiva, entendida como:

\section{La relación de los sujetos con la cultura que habita, los rasgos del lugar que ocupa en so- ciedad, las diferencias de su capacidad crea- tiva, de la autonomía de su imaginación, del ejercicio de su libertad para orientar las deci- siones de su vida (Galende, 2015, p. 16).}

Se constata que el campo de la salud mental está más bien dominado por exigencias de gestión y estandarización, excluyendo así la dimensión subjetiva de los modos de comprender y tratar el sufrimiento psíquico (Castro-Sardi, 2016).

Por lo tanto, se asume en este estudio una perspectiva que permita comprender los modos de relación entre el sujeto y el emprendimiento y los recursos subjetivos con los que cuenta para asumir un proyecto económico. Este último puede tener diversos sentidos en cada proyecto de vida y no se restringe únicamente a un medio para generar ingresos económicos. Se propone una mirada que tenga en cuenta "la singularidad de cada sujeto, su territorio de vida, su historia y las condiciones reales de su existencia" (Galende, 2015, p. 16). Con este propósito se acude al psicoanálisis aplicado, de orientación lacaniana, que propone una teoría de la subjetividad, que considera superflua la distinción entre lo psíquico y social, porque "el sujeto del psicoanálisis es impensable sin referencia al vínculo con el otro 
y a su lazo de sujeción con el lenguaje" (CastroSardi, 2014, p. 119). El sujeto existe en tanto es nombrado por los otros, está inscrito en una genealogía y en unos lazos de parentesco y, por lo tanto, su existencia es social, incluso antes de su nacimiento biológico (Peláez-Jaramillo, 2011; Castro-Sardi, 2014). La importante determinación inconsciente de los actos subjetivos de ninguna manera exime al sujeto de la responsabilidad frente a sus decisiones y acciones; incluso, el método psicoanalítico propone lo que Miller (2003) ha denominado "rectificación subjetiva" para designar la implicación y responsabilidad de un sujeto frente a sus dichos y actos.

La aproximación de la subjetividad que propone el psicoanálisis introduce una diferenciación entre el sujeto y el yo de la conciencia y la razón, en la medida en que se reconoce la dimensión inconsciente de la vida psíquica. "El sujeto está descentrado del yo que habla, no es ni el yo que habla, ni el yo que piensa, y aunque el yo está en escena, tampoco representa al sujeto, el cual le es desconocido" (Peláez-Jaramillo, 2011, p. 5). Lo "desconocido" es lo que Freud denominó inconsciente, del cual dan cuenta ciertos fenómenos de la vida cotidiana, como son los sueños, lapsus, actos fallidos, chistes y síntomas (Castro-Sardi, 2014). Desde esta perspectiva, se reconoce también que las decisiones de los sujetos no son siempre transparentes. La intencionalidad de los actos, las motivaciones y los deseos no son siempre claras para los sujetos, porque el deseo es inconsciente: muchas veces las personas no saben lo que quieren y no pueden diferenciar sus deseos de aquello que desean los otros. El deseo es una relación siempre dialéctica y parte de una pregunta: ¿qué deseo? Y la búsqueda de una respuesta siempre remite al Otro: ¿qué quiere el otro de mí? Pero la respuesta del Otro nunca es directa, ni transparente, porque está marcada por una falta de saber y una falta de objeto que satisfaga al sujeto plenamente. En palabras de Bueno-Restrepo (2012):

El deseo es movimiento, es un desplazamiento metonímico a través de objetos que imaginariamente lo habrían de colmar; el deseo permite que la libido invista los objetos del mundo, los de la fantasía, y que no se produzca su estasis en el cuerpo; el deseo comporta la falta, el vaciamiento de goce" (p. 10-11).

El deseo se opone así al goce, otra forma de nombrar la pulsión de muerte propuesta por Freud (2004), aquello que se contrapone a la vida y que habita todo sujeto. El goce se hace presente, por ejemplo, en las relaciones y circunstancias de sufrimiento que solemos repetir de modo inconsciente (Bueno-Restrepo, 2012). El goce, en oposición al deseo, es inmovilidad, petrificación, sufrimiento y se manifiesta en los síntomas. El deseo es lo que moviliza al sujeto a iniciar y persistir en sus proyectos de vida (De Castro-Korgi, 2011). Se considera, en el contexto de esta investigación-intervención, como un recurso subjetivo que permite encontrar y reencontrar las razones propias para emprender, tomando distancia del deber ser o de las expectativas sociales que vienen como imperativo del Otro.

Por consiguiente, el psicoanálisis hace una apuesta por la subjetividad y por la posibilidad que tiene todo ser humano de dar cuenta, mediante la palabra, de aquello que desconoce de sí mismo, tanto de su deseo como sus elecciones sufrientes de repetición. En palabras de Gallo (2011): "aquí el dicho vale por lo que revela del ser que sufre, allá por lo que pone al descubierto, así sea entre líneas, acerca de la implicación del sujeto en fenómeno en cuestión" (p. 109). La cuestión del sufrimiento, en tanto ligado a la experiencia humana, remite siempre a una "dificultad en la relación con el Otro, llámese desinserción, exclusión o precarización del lazo social" (Castro-Sardi y Munévar, 2016, p. 23). Diversos autores han introducido el fenómeno de cómo, en la actualidad, tenemos cada vez más dificultades para entablar lazos sociales (Galende, 2008). Esta dificultad se torna más aguda en los contextos de exclusión social, debido al intento de invisibilización de los "residuos humanos", que nombra Bauman (2005), como una de las características de la modernidad tardía. En la cual se tiende a segregar y desechar al otro, construido como diferente y amenazante para el orden social.

Ante estas nuevas realidades de sufrimiento social, Bauman (2005), y otros autores, hacen un llamado a buscar soluciones locales a problemas 
generados por la economía globalizada. Es en este marco que el CAPsi propone un modelo de atención en salud mental que reconoce la dignidad y autonomía, como una forma de empoderar a los sujetos sobre sus circunstancias; y propone un abordaje del malestar subjetivo por medio de la escucha y la palabra. Así mismo, se orientan los procesos de intervención situando las coordenadas sociales y culturales de los sujetos y privilegiando las soluciones singulares que inventan las personas frente a sus circunstancias. Lo que implica dejar de lado las propuestas universalizantes y padronizadas de tipo "para todos" (Castro-Sardi, 2013; 2016).

\section{Método}

Este estudio tiene un enfoque de investigación social cualitativa, el tipo de análisis de datos es interpretativo. El proceso anterior es apoyado por elanálisis hermenéutico del resultado estadístico descriptivo de los datos socioeconómicos de los sujetos, y de un cuestionario estructurado aplicado a los mismos. Una metodología cualitativa permite la comprensión de la realidad como una producción sociocultural, en la que se priorizan los sentidos y significados que tiene, para el sujeto, su experiencia en la vida cotidiana, y su aporte a esa realidad (Galeano, 2004; González-Rey, 2006). Esta perspectiva visibiliza el lugar singular del emprendimiento en la vida de las mujeres inscritas en el PCE, los recursos subjetivos y sociales que tienen para afrontar esa actividad, y su fortalecimiento a partir de un espacio de escucha y orientación psicosocial.

\subsection{El marco institucional de investigación-intervención}

El proceso se realizó con las beneficiarias de un PCE ofertado por una institución que cuenta con una trayectoria de tres décadas apoyando a las mujeres microempresarias. Este PCE va dirigido prioritariamente a mujeres provenientes de contextos económica y socialmente vulnerables, ofreciendo módulos de capacitación en emprendimiento, educación financiera, alfabetización digital y liderazgo. El espacio de escucha psicosocial (estrategia de atención en salud mental) se ofreció a las beneficiarias que voluntaria y libremente demandaran el servicio.

\subsection{Participantes}

La muestra utilizada para el estudio fue de 32 beneficiarias de la estrategia de atención de salud mental, vinculadas al PCE. Fueron seleccionadas de un universo de 95 beneficiarias que consultaron por problemas de salud mental, con base en el criterio de adherencia al proceso de atención psicosocial (asistencia mínima de 4 sesiones). Todas las participantes dieron su consentimiento por escrito, una vez recibieron la información sobre los fines de la investigación y el manejo de la información.

Sobre la caracterización de la muestra: 9\% (3) hombres y $91 \%$ (29) mujeres; con un promedio de edad de 43 años; $100 \%$ de los participantes se reconocían como mestizos; 3\% (1) desplazados del conflicto armado; $22 \%$ (7) han sufrido episodios de maltrato; $62,5 \%$ (20) consultaron por problemas de pareja; $19 \%$ (6), por crisis de identidad; $6,25 \%$ (2), sufren de enfermedades mentales crónicas; $6,25 \%$ (2), consultan por conflictos familiares; $3 \%(1)$, por consumos adictivos de sustancias; y 3\% (1), por problemas laborales.

Estos participantes provienen de una población universo (95) con las siguientes características: $77 \%$ (73) fueron mujeres y el $23 \%$ (22) hombres; promedio de edad: 45 años; $77 \%$ (73) se identifica como mestizas; $20 \%$ (19), como afrocolombianas; y $3 \%$ (3), como indígenas. $9 \%$ (9) ha sido víctima del conflicto armado. $46 \%$ (44) de la población ha sufrido algún tipo de violencia (doméstica, armada, sexual, urbana o escolar). $42 \%$ (40) de los consultantes provienen del Distrito de Aguablanca; 22\% (21), de municipios aledaños; y 36\% (34), de la zona centro, norte y ladera de la ciudad de Cali. En términos de problemáticas de salud de mental, las principales razones de consulta fueron: $35 \%$ (33) por problemas de pareja; $14 \%$ (13), crisis de identidad; y $14 \%$ (13), enfermedad mental. 


\subsection{Instrumentos}

- Ficha socio-económica: se compone de información socio-demográfica, como edad, nivel educativo, estado civil, número de personas a cargo y reconocimiento étnico y datos económicos, como tipo y tenencia de vivienda, ingresos, subsidios, y otras ayudas económicas.

- Registro del proceso terapéutico (RT): se compone de los datos del psicólogo y supervisor; los datos socioeconómicos del consultante; notas del proceso terapéutico (motivo de consulta, fecha de inicio y terminación, principales intervenciones terapéuticas y logros); y las recomendaciones de la supervisión del caso.

- Cuestionario estructurado (aplicación pre y post): instrumento diseñado a partir de algunos ítems relacionados con problemáticas psicosociales de la escala de bienestar psicológico de Ryff (Díaz et al., 2006), y preguntas relacionadas con la actividad de emprendimiento. La aplicación "pre" se realizó en primera cita, mientras que la aplicación "pos" se realizó después de la última cita.

- Atlas-ti: fue el software utilizado para analizar las notas de los registros terapéuticos de los y las consultantes.

\subsection{Procedimiento de intervención}

Se ofertó un modelo de atención psicosocial de duración limitada, gratuita, dirigida a los estratos sociales socioeconómicamente vulnerables (Castro-Sardi y Munévar, 2016). Este modelo se adaptó a las necesidades de las beneficiarias del PCE, adicionando actividades grupales que tenían la finalidad de informar sobre la existencia del servicio de atención psicológica y mitigar las barreras de acceso al servicio; un módulo de capacitación en salud mental, habilidad de escucha y remisión responsable para el equipo de facilitadores y formadores del PCE. La atención psicológica individual y familiar se ofrecía semanalmente con una duración promedio de 40 minutos la sesión, y con la posibilidad de extender el proceso de atención según las necesidades del caso. También se habilitó un servicio de cercanía con el fin de eliminar las barreras de acceso relacionadas con la movilidad en la ciudad. En el transcurso de 11 meses, se atendieron 95 personas. A continuación, se presenta en la Figura 1 el proceso de atención psicosocial de la estrategia de salud mental.

Figura 1. Proceso de atención psicosocial

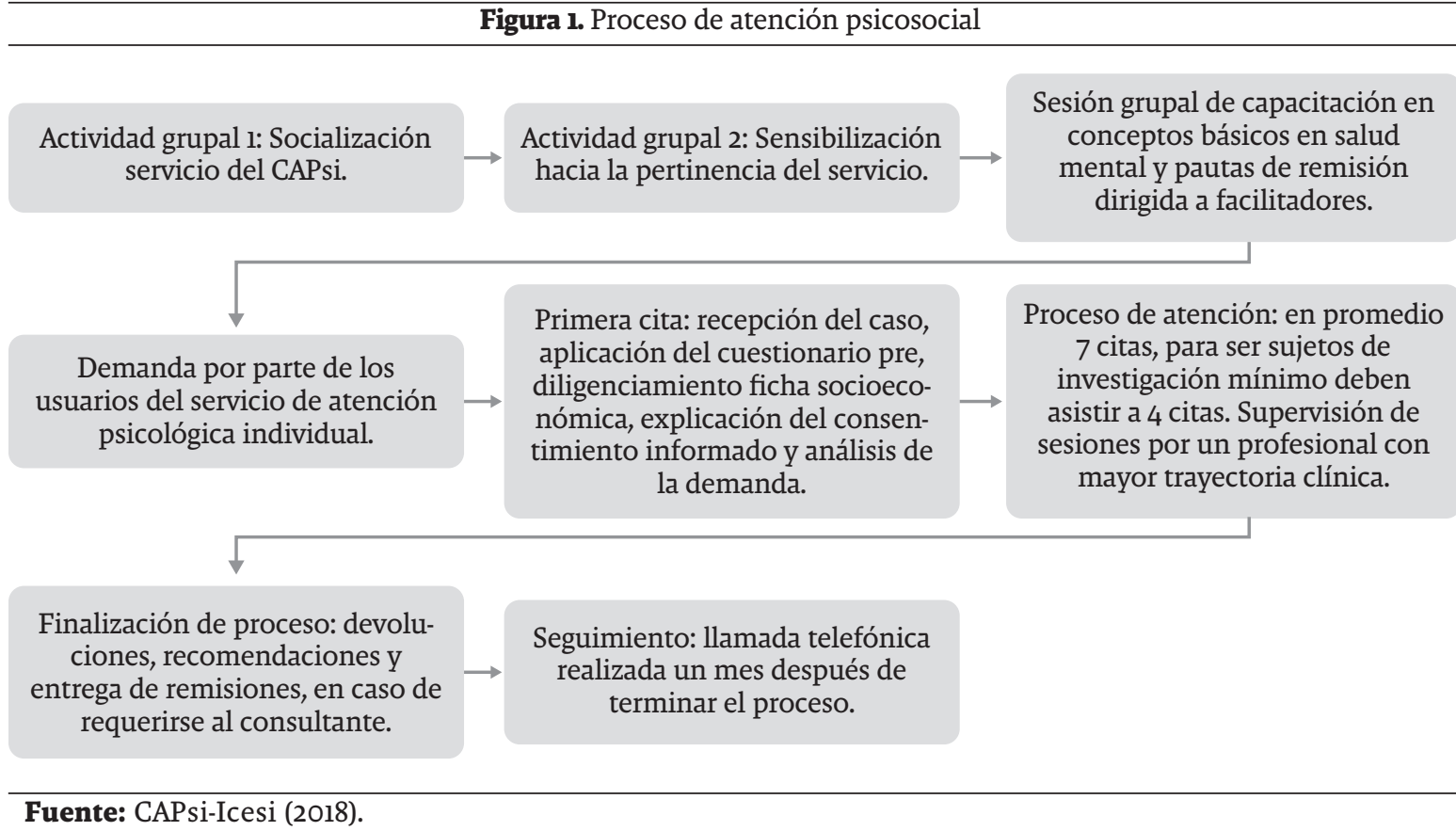




\subsection{Procedimiento de investigación}

Se realizó un análisis hermenéutico interpretativo de los registros terapéuticos de 32 beneficiarias, lo que equivale a 131 sesiones individuales de atención, con el objetivo de encontrar los significantes asociados a las categorías definidas a priori, sin dejar de lado los significantes emergentes, que pasaron a formar nuevas categorías (Ángel, 2011; Castillo y Gómez, 2004). Los datos anteriores se apoyaron con la interpretación de los resultados del análisis estadístico descriptivo de los datos sociodemográficos de toda la población de intervención (95 personas) y de los datos del cuestionario estructurado pre y post aplicado a las 32 personas. El software ATLAS.ti se utilizó para el análisis de los registros terapéuticos.

\subsection{Categorías de análisis}

Las categorías de análisis para los registros terapéuticos fueron las siguientes:

- Problemáticas psicosociales: aspectos psicosociales que impiden o hacen difícil para el sujeto emprendedor llevar a buen término su actividad emprendedora.

- Emprendimiento: "cualquier intento de creación de un nuevo negocio o una nueva empresa, tales como el autoempleo, una nueva organización comercial, o la expansión de un negocio ya existente, por un individuo, un equipo, o un negocio ya establecido" (GERA, 2018).

- Empoderamiento: "la expansión de las habilidades de las personas para tomar decisiones estratégicas para sus vidas en condiciones en las cuales estas habilidades han sido rechazadas previamente" (Kabeer, 1999, p. 437).

- Recursos subjetivos: las herramientas que el sujeto utiliza para construir una solución posible a ese malestar, son las formas de saber-hacer con su sufrimiento.

- Efectos y logros terapéuticos: verbalizaciones de las consultantes que den cuenta de una mejoría de su situación y de cambios en su posición subjetiva frente a un problema.

\section{Resultados}

El proceso de análisis de entrevistas permitió identificar las problemáticas psicosociales más comunes en las consultantes y los efectos de la atención psicosocial en las mismas, además de la relación entre condiciones mentales crónicas, emprendimiento y atención psicosocial. A continuación, se presentan las categorías construidas a partir de este análisis basado en frecuencias y ocurrencias:

\subsection{Problemáticas psicosociales}

\subsubsection{Mujeres desbordadas de responsabilidades: La mujer que se "hacía cargo de todos"}

Quince (15) de las historias clínicas analizadas dan cuenta de mujeres desbordadas por las responsabilidades (lo que equivale al $52 \%$ de la población femenina). Estas se refieren a las obligaciones del hogar y cuidado de su familia extensa, a lo cual se adiciona la función como empresarias. Una de las consultantes lo expresa de la siguiente manera:

[Yo] era la que "hacía todo" entonces los otros se "relajaban"... [Ella] es "la que corre, la que hace" (RT-597).

En los cuestionarios de bienestar psicológico, $72 \%$ (20 mujeres y 3 hombres) manifestaban que la falta de "apoyo familiar" era una de las dificultades más importantes en su actividad emprendedora. Esta problemática habla de mujeres que asumen muchas responsabilidades y delegan poco.

\subsubsection{Experiencias de maltrato: “Me enseñaron que había que mantener el hogar", "había que aguantarse"}

Otra temática común en los registros terapéuticos es la vivencia de experiencias de maltrato, más común en mujeres: 18 de estos casos fueron vividos en el pasado en relaciones con sus ex parejas o parejas actuales. En el cuestionario, 40\% (19 mujeres y 1 hombre) manifiestan que han sufrido algún tipo de maltrato durante 
su vida. Respecto a violencia sexual, 5 personas, todas mujeres, narran sucesos de este tipo en sus historias clínicas. Una de las consultantes logra historizar sus relaciones de maltrato de la siguiente manera:

\begin{abstract}
[La psicóloga señala que la consultante permaneció mucho tiempo con un hombre maltratador, ante lo cual la consultante responde] (su familia le había dicho) "que había que aguantarse, porque mi padre también fue un hombre maltratador con $\mathrm{mi}$ madre, jsupuestamente era así!” (RT-597).
\end{abstract}

\subsubsection{Dificultad para la toma de decisiones: "no sé qué hacer", "me siento cansada", "me siento estancada"}

Las principales problemáticas que les generan conflicto a los consultantes, respecto a su emprendimiento, se encuentran relacionadas con la distribución de funciones (porque ellas asumen roles diversos además del de emprendedoras); negociación de prioridades en sus socios y la definición del enfoque de su emprendimiento. Este último se refiere a la dificultad para enlazar su propio interés con su proyecto de emprendimiento y se produce cuando el emprendimiento proviene de los intereses y habilidades de la persona, pero su entorno familiar o social no apoya el desarrollo del mismo; y/o cuando el emprendimiento ha surgido como una idea familiar, pero la persona no se identifica con este.

En el cuestionario, el 20\% (6 mujeres y 1 hombre) de los consultantes manifiestan que la toma de decisiones representa algún grado de dificultad en su emprendimiento, como se puede apreciar a continuación desde la historia clínica de la consultante:

El negocio de las obleas es muy bonito, es mío, pero sin poder opinar al respecto. $[\mathrm{Al}$ final dice que si no la llaman para este negocio] me quedaré con el de bisutería que sí me gusta, además con la tienda que ya tengo en mi casa. [Aunque una de las debilidades que le encuentra a este último] es que no he podido surtirlo y en ocasiones cuando no hay para el mercado, tomamos productos de ahí, nos comemos el negocio (RT-617).

La toma de decisiones como dificultad es un tema transversal en las 32 historias clínicas analizadas, y en la vida personal tiene que ver con las relaciones sentimentales, en las que hay casos de maltrato o abuso; la delimitación de funciones y responsabilidades en el contexto familiar; y los cambios de hábitos y consumos adictivos de sustancias.

\subsubsection{Dificultades en el establecimiento del lazo social: "da miedo hablar con los clientes, gerentes, gente desconocida, son personas muy importantes"}

Algunos consultantes manifiestan que tienen dificultad para relacionarse con otras personas, exponer sus puntos de vista o desempeñarse en situaciones sociales desconocidas por temor a fallar, lo que hace difícil el relacionamiento con clientes internos y externos de su actividad emprendedora. Una de las consultantes narra sus problemas para establecer lazos sociales en el contexto de su negocio de la siguiente manera:

\section{La consultante se siente muy dependiente de su madre... cuando tiene que ofrecer sus servicios sobre el pulido de pisos, le pide mejor a su madre que lo haga, pues no se siente capaz de hacerlo (RT-633).}

Esto también se refleja en el cuestionario de bienestar psicológico, en el que $84 \%$ (25 mujeres y 2 hombres) de las personas manifestaron que era necesario fortalecer su capacidad para aceptarse positivamente a sí mismas.

\subsection{Recursos subjetivos identificados y efectos terapéuticos}

\subsubsection{Efecto terapéutico 1: Reconocer y dar importancia al propio deseo}

En 15 de las historias narradas por las mujeres se aprecia una dificultad para poner un límite a las demandas del otro. Estas mujeres 
tienden a supeditar sus deseos, sus necesidades, sus sueños, etc., a los requerimientos de sus familias, negocios, círculos sociales, entre otros.

Como resultado del proceso de acompañamiento psicosocial, se logró que las consultantes identificaran sus propias capacidades, y que dieran validez a sus razones para negociar la demanda de los otros en favor de su deseo, y no necesariamente satisfacer siempre a los demás.

Otro efecto terapéutico se relaciona con que las consultantes comenzaron a delegar funciones en sus hogares y en sus negocios, lo que dio lugar al empoderamiento en ellas, para rechazar o negociar responsabilidades que no necesitaban o no deseaban asumir. En el cuestionario, el 52\% (11 mujeres y 1 hombre) reportó una disminución en la percepción de falta de apoyo familiar como una barrera en su proceso de emprendimiento. Una de las consultantes, al final de su proceso, expresa el cambio de la siguiente manera:

La consultante dice que ahora se está preocupando más por ella y por sus necesidades, porque dice que antes era como la madre de todos. La terapeuta le resalta que ella se ubicaba en esta posición, ante lo cual la paciente responde que veía las necesidades de los demás y quería ayudar en eso. "Pero he aprendido a decir no, ahora me preocupo más por mí misma” (RT-597).

\subsubsection{Efecto terapéutico 2: construir una posición de responsabilidad frente a las situaciones de maltrato}

En 5 casos de violencia doméstica, el proceso de atención psicológica contribuyó a construir una nueva posición de las mujeres frente a la situación de maltrato. Esto se logró por medio de recursos subjetivos como reconocer el coraje de las decisiones tomadas, y las reivindicaciones económicas y sociales que estas mujeres han logrado.

También se presentaron efectos terapéuticos en los casos de nueve mujeres que en el pasado habían experimentado situaciones de maltrato. La intervención se enfocó en albergar el sufrimiento y las secuelas que estas situaciones habían ocasionado en ellas, y resaltar la decisión que habían tomado de poner punto final a esas relaciones.

En los tres casos en los que las consultantes aún conviven con sus parejas -e indicaron que, en ese momento, ya no había maltrato físico-, se optó por historizar las relaciones; de tal forma que ellas pudieran identificar los mecanismos de dominación por parte de sus parejas y cómo han cambiado con el tiempo, pasando de ser agresores físicos a ser agresores pasivos. Principalmente por medio de mecanismos como no asumir responsabilidades en el funcionamiento del hogar, del negocio e incluso de ellos mismos. Este proceso contribuyó a que las consultantes construyeran una posición más crítica ante esta falta de responsabilidad del otro.

\subsubsection{Efecto terapéutico 3: Fortalecer la confianza en sí mismos/as}

En cuanto a las y los 27 consultantes que se acercaron con demandas relacionadas con fortalecer la seguridad en sí mismas ( 25 mujeres y 2 hombres), el proceso hizo énfasis en resaltar, como recursos subjetivos, los logros y las capacidades que estas personas tenían y que, sin embargo, no habían sido lo suficientemente reconocidos. Una de las consultantes comienza a cuestionarse sobre el hecho de que su trabajo tiene un valor que debe ser reconocido:

\footnotetext{
La consultante comenta que "le da pena ofrecer sus productos, cobrarle a la gente, que le digan que es caro". Ella menciona "que es así porque ella realiza todo a mano", la terapeuta le resalta que ese es el valor de su trabajo. La consultante se pregunta a sí misma, si ella "puede, si vale la pena" (RT-596).
}

Por otra parte, también se contribuyó a disminuir las expectativas que los consultantes ubican en sí mismos, y que, al no lograrlas, generan sentimientos de culpa que se traducen en inseguridad. 


\subsection{Efectos a nivel del empoderamiento y emprendimiento}

\subsubsection{Toma de decisiones respecto al negocio}

En el análisis de las historias clínicas se encontró que $63 \%$ (20) de las personas experimentaron efectos positivos en sus procesos de emprendimiento. Mientras que 37\% (12) de las personas no manifestaron cambios significativos en sus negocios.

El principal recurso subjetivo que permitió movilizar este logro terapéutico fue la validación de las propias capacidades, por medio del cual se contribuyó a que las y los consultantes:

- Identificaran opciones sobre el enfoque que debería tener su negocio, ligando su deseo con el mismo, ya que en algunos casos ellas no se sentían identificadas con su emprendimiento.

- Construyeran una imagen de sí mismas basada en la confianza en las propias capacidades para llevar a buen término sus ideas de negocio, resaltando los logros que han tenido al respecto.

- Aprendieran a direccionar adecuadamente relaciones humanas que estaban minando su capacidad para enfocar asertivamente sus acciones en su negocio.

En términos de los efectos de la atención psicosocial en la actividad emprendedora de los/las consultantes, estos principalmente fueron a nivel del proceso organizacional del negocio, específicamente el mejoramiento en términos de relaciones laborales, capacidades de negociación, trabajo en equipo y toma de decisiones. Una de las consultantes lo expresa de la siguiente manera, respecto a la distribución de funciones con su esposo:

\footnotetext{
Tenía una intención con esto [se refiere un viaje al que se fue sola, sin su familia] y era de forzar a mi esposo a que se pusiera en mi lugar... Le dejé toda carga a él, de lo que yo hacía. Porque me di cuenta que los otros también pueden hacer las cosas, solo fue cuestión de permitirlo (RT-563).
}

\subsection{El emprendimiento como modo de inserción social}

\subsubsection{El acompañamiento para el emprendimiento como red de apoyo}

Respecto al rol del acompañamiento del PCE, por medio de su programa de emprendimiento, las personas lo perciben como un espacio que ofrece: "apoyo", "aprendizaje", "(lugar donde se siente) muy bien, (porque) ve gente nueva", "un empujón". Respecto al proceso de formación, hacen referencia al curso de liderazgo, donde han logrado identificar situaciones personales que les afectan y les han permitido darse cuenta de que existen soluciones posibles a los mismos. En cuanto a esto último, es importante resaltar que, en algunos casos de quienes solicitaron consulta o fueron remitidos, las intervenciones grupales generaron demasiado impacto emocional, situación que se salía de las posibilidades de atención del dispositivo de formación. 16\% (5) de las personas realizaron alusiones explícitas sobre este hallazgo.

\subsubsection{Cronicidad mental e inserción social a través del emprendimiento}

Durante el transcurso del proceso de atención, se identificaron 14 personas con condiciones crónicas de salud mental. Todos se encuentran estabilizados gracias a su red de apoyo familiar e institucional, lo que incluye a la institución que ofrece el PCE. Sin embargo, es importante resaltar que estas personas pueden ser especialmente sensibles a las situaciones de presión personal o social del entorno, que cuando no son manejadas adecuadamente pueden contribuir a desencadenar una crisis de salud mental. Uno de los emprendedores, que presenta un estado de cronicidad mental, expresa esta disyuntiva así:

\footnotetext{
El consultante narra con angustia que no estaba de acuerdo con hacer uso de un obsequio que se le proporcionó al terminar el proceso de formación para tomarse una foto. Manifiesta que se sintió forzado
} 
a hacerlo, fue algo incómodo para él... sintió que todos tenían que ser iguales, haciendo lo mismo, como estar con actitud positiva y con eso no está de acuerdo (RT-598).

A continuación, se presenta la Figura 2, que resume los resultados de investigación narrados en esta sección.

Figura 2. Esquema de resultados de investigación

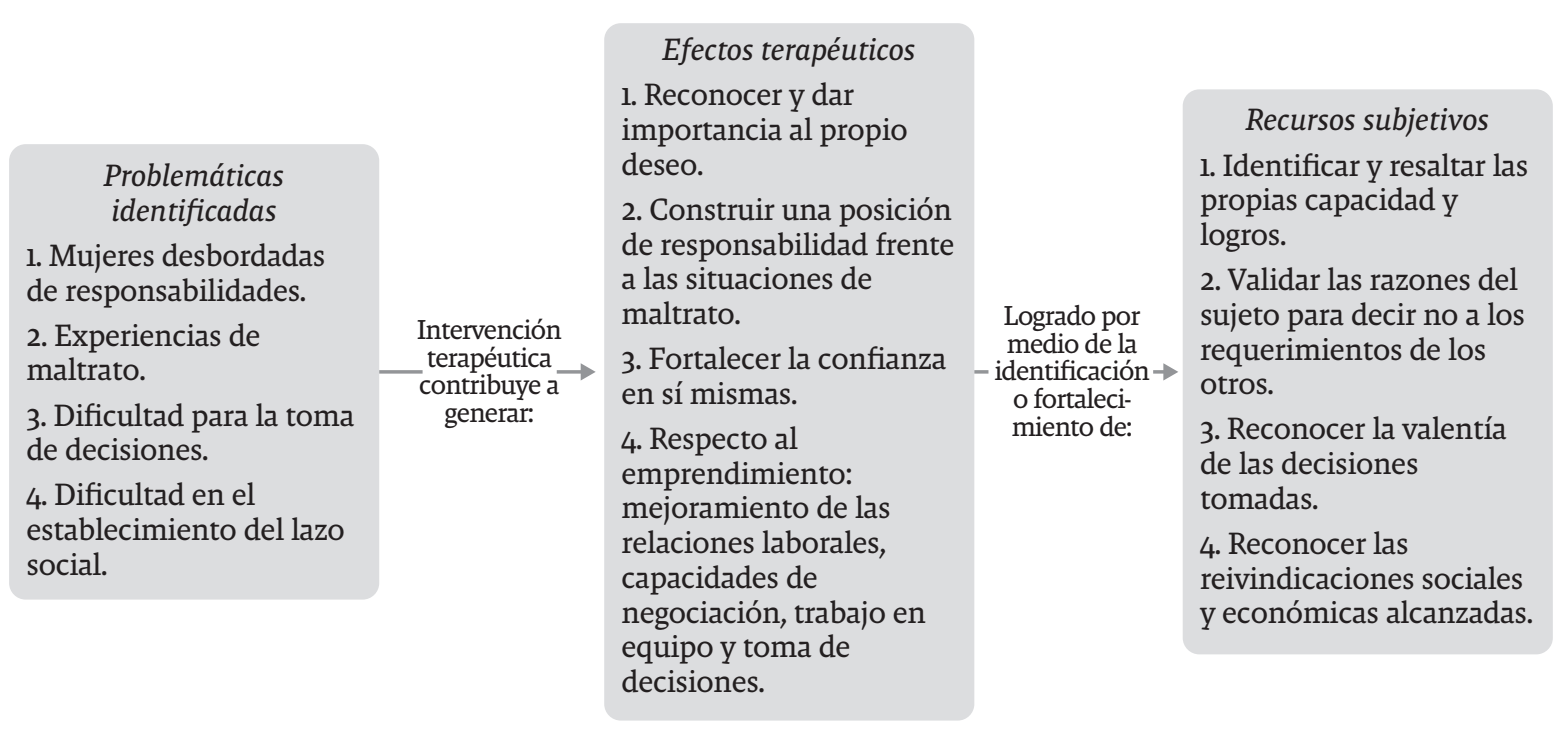

Fuente: CAPsi-Icesi (2018).

\section{Discusión}

El proceso de acompañamiento en salud mental permitió evidenciar una serie de paradojas sobre la forma como se ha configurado la relación entre los/las sujetos que participaron en este estudio y la actividad emprendedora.

\subsection{Paradoja 1: Emprendedora exitosa - mujer subordinada}

Como se ha citado anteriormente, el fortalecimiento económico, por medio del emprendimiento, no necesariamente es causa suficiente para promover procesos de empoderamiento femenino, como lo expresan Kabeer (1999) y Rowlands (1997). Esto se evidenció en las mujeres del presente estudio, ya que en algunos casos se encontró que, aunque eran propietarias de emprendimientos con altos niveles de productividad, vivían condiciones de subordinación e incluso de maltrato por parte de sus parejas. El estudio realizado por Annan et al. (2015), sobre emprendimiento y violencia de género, concluye que el emprendimiento puede contribuir a aumentar los niveles de ingreso, capacidad adquisitiva o riqueza, pero el "empoderamiento es más escurridizo" (p. 187). También concluye que aumentar el ingreso económico no tiene ningún efecto en la violencia ejercida por su pareja íntima un año después del proceso. Por el contrario, puede ocurrir que este incremento contribuya a aumentar las posibilidades de violencia de género.

Sobre la dificultad de las mujeres para ubicarse como agentes de decisión o empoderadas, también se debe pensar desde las lógicas actuales de la sociedad, que se rigen por valores que "son una expresión de una nueva hegemonía social, detentada por las formas de individualidad propias de los valores de mercado que dominan al mundo" (Galende, 2008, p. 220). Asimismo, la individualización va promoviendo la fractura de los lazos sociales, dado que se espera que 
autónomamente la persona asuma un lugar en la jerarquía social, introduciendo así un principio de competencia (Galende, 2008). Este principio de competencia afianza la fragmentación de los lazos sociales, lo cual tiene efectos en la subjetividad de las personas. Por ejemplo, en los casos de las mujeres maltratadas se ve una mayor fragilidad para asumir lugares sociales, como la familia, mayores cargas de sufrimiento mental, y la dificultad de poder ubicar los recursos para hacer frente a los conflictos.

Frente a esta problemática, desde la estrategia de atención psicosocial en salud mental, se promovió la "rectificación y responsabilidad subjetiva" (Miller, 2003, p. 69), abriendo un espacio de escucha y auto-reflexión para que cada una se preguntara por su lugar en su familia, más allá del éxito en su empresa. Una vez identificadas las posiciones de subordinación y las circunstancias de maltrato, se les acompañaba en la construcción de salidas y soluciones considerando sus contextos relacionales particulares. Si bien se logró que en algunos casos se generaran cambios significativos en el posicionamiento de las mujeres que sufrían de condiciones de maltrato, se constató que estos procesos de cambio subjetivo toman más tiempo y requieren de una red de apoyo familiar e institucional sólida que no siempre estaba presente.

\subsection{Paradoja 2: La mujer omnipotente - la mujer sacrificada}

En la actual sociedad se han establecido nuevos valores de inscripción social, y uno de ellos es el imperativo de la felicidad. El cual no se basa en el deseo, como una pregunta singular, sino que se da el imperativo de mostrarse feliz, que se puede todo, a partir de tener lo que el otro tiene (Holguín, 2012); como en el caso de algunas participantes del estudio. Por consiguiente, los sujetos ya no se movilizan por un deseo de ser madre o de amar a una persona, sino que se rigen por el hecho de sostener un ideal, como es el de la familia nuclear, que incluso puede ser a costa del sujeto mismo, en palabras de las consultantes: "había que aguantarse". Este dicho da cuenta de la posición de goce, de satisfacerse en su propio dolor por sostener una relación de maltrato, lo cual no exime la responsabilidad de las personas que maltratan o cometen abusos. $\mathrm{El}$ hecho de regirse por imperativos tiene como efectos la alienación del sujeto a los mandatos del Otro, por lo que hace más difícil que el sujeto encuentre los elementos que le permitan tomar la vía del deseo, y pueda asumirse como un agente de decisión.

Estas cuestiones también son abordadas por Jaramillo (2013), en su texto sobre los obstáculos subjetivos que enfrentan las mujeres para realizar un goce efectivo de sus derechos. La autora encuentra, a partir de sus entrevistas y trabajo de campo, que "el sacrificio" es una posición asumida por muchas mujeres, en el que "se da prevalencia al bienestar y logros del otro... a menudo... dicho sacrificio da lugar a un sufrimiento que pudiera evitarse" (p. 68). Esta es la posición de un número importante de las mujeres del proceso de investigación: se podría pensar que se ha entendido el empoderamiento no desde la posibilidad de situarse en una posición de poder, desde la cual se negocia con el otro y se decide en igualdad de condiciones, sino desde el imperativo de hacerse cargo de todo, de decir "si" a todo. Aun cuando esto genere sufrimiento y malestar. Ante esto, la atención psicosocial logró, en algunos casos, el cuestionamiento, por parte de cada una, de su propia posición sacrificial y, en la medida en que ellas mismas identificaban las decisiones que las habían conducido a sus circunstancias actuales, también podían tomar decisiones a través de las cuales lograban poner límites, delegar funciones y negociar responsabilidades con el otro (parejas, socios, colegas).

Es pertinente, en este punto, interrogarse sobre la promoción del emprendimiento como fórmula universal para lograr el empoderamiento femenino. Si bien es cierto que, en muchos casos, abre el camino de la emancipación, en otros puede producir el efecto contrario. Por lo anterior, es necesario reflexionar permanentemente sobre los efectos de las intervenciones sociales mediante el emprendimiento, de tal manera que no se promuevan "nuevas formas de esclavitud para las mujeres, porque ya no deberán ser excelentes madres y esposas, sino 
además, excelentes trabajadoras" (Jaramillo, 2013, p. 74).

\subsection{Paradoja 3: El emprendimiento para la inclusión o exclusión social}

La erradicación de la pobreza es el primer objetivo de la agenda de los Objetivos de Desarrollo Sostenible-Agenda 2030 y se considera que la inclusión social y la equidad de género son aspectos claves (Johansson y Sjindjapkin, 2015, p. 8). Al mismo tiempo, se considera que una vía para promover esos aspectos claves es la búsqueda del crecimiento económico y que éste, a su vez, debe buscar la inclusión social y económica de las poblaciones, para lo cual, como hasta el momento se ha apreciado, el emprendimiento ha sido considerado como un mecanismo privilegiado de acción.

En esta investigación-intervención se encontró que, efectivamente, el PCE contribuye a la inclusión social y económica de sujetos, en la medida en que las personas encuentran un espacio de "apoyo" para sus capacidades y la superación de algunas de sus problemáticas. Es importante resaltar que, para sujetos con condiciones mentales crónicas, los facilitadores y funcionarios del PCE constituyen una red de apoyo que los estabiliza y les permite ser funcionales e insertarse en la sociedad.

Sin embargo, la búsqueda de la inserción social deja en su camino procesos de exclusión social, lo cual no solo se relaciona con los programas de emprendimiento, sino con los procesos de intervención social en general (Dolan y Rajak, 2016). En este estudio se encontró que algunas personas no sentían que la forma como se implementa el programa de emprendimiento respondiera a sus necesidades, lo cual se relacionaba con el proceso de estandarización de la intervención. Uno de los sujetos expresa que no todos deberían ser considerados iguales, refiriéndose a las intervenciones estandarizadas; en otro caso, una mujer expresa sentirse "desanimada", debido a que se siente obligada a asumir un proyecto de negocio que no le interesa, pero que le es impuesto desde lógicas externas de financiación.

Los casos anteriores no son la generalidad del proceso de intervención, pero muestran cómo las intervenciones sociales no deberían reducirse a un protocolo para estar atentos a escuchar los elementos subjetivos que se escapan a los estándares o a los procesos preestablecidos (Desviat, 2015). Esto remite a que el interventor social se pregunte por la ética de su intervención, en lo que respecta a los efectos que genera la intervención como respuesta a la demanda de un sujeto la responsabilidad del interventor en relación a las circunstancias de la relación, y el objetivo real de la intervención y de los mecanismos que se implementan en el proceso (Moreno, 2013).

A través del proceso de atención psicosocial, y a partir de las problemáticas expresadas por los beneficiarios, se buscó introducir los aspectos subjetivos, mediante la escucha de la singularidad del sujeto (Castro-Sardi, 2016); y, en algunos casos, se logró enlazar de una forma particular el deseo del sujeto al proceso de emprendimiento. Así como también, en otros casos, ciertas personas comenzaron a cuestionarse si el emprendimiento era un camino que deseaban recorrer.

En cualquier caso, surge la pregunta sobre las intervenciones sociales, los intereses que sirven y el efecto que tienen en las personas en contextos de alta vulnerabilidad social. Es común escuchar las historias de éxito en los procesos de emprendimiento, así como en otras intervenciones sociales que promueven la inserción socio-económica. Pero, ¿sabemos qué sucede con aquellos que desertan? ¿Qué ocurre con aquellos que no culminan exitosamente sus negocios? Sigue siendo un desafío pensar en formas de inclusión de las poblaciones más vulnerables y frágiles en los procesos de intervención y desarrollo social. Desde nuestro punto de vista, es urgente atender a las necesidades particulares, tener en cuenta las experiencias e historias subjetivas, y no presuponer una única vía de inclusión social. 


\section{Conclusiones}

Colombia es uno de los países con mayor número de emprendedores, pero con menor impacto económico y social del emprendimiento (Eslava, 2019). Lo anterior es tendencia general en la región de América Latina y el Caribe: un gran número de emprendimientos, poca innovación, poco impacto, poca supervivencia (Lederman et al., 2014). Frente a este contexto, y a partir de la discusión y resultados de este artículo, centrados en gran parte en la importancia de la singularidad del sujeto emprendedor, es pertinente la pregunta por ¿cuáles son los elementos necesarios para lograr procesos de inclusión socio-económica real y efectiva por medio de los programas de emprendimiento? Al respecto, para el caso colombiano, Piedrahita (2019) afirma que "No todo el emprendimiento es igual. Y trazar y ejecutar políticas y planes potentes para estimularlo requiere tratar de manera diferente lo que es distinto" (p. l). El autor hace referencia a que, desde la política de desarrollo económico, se deben tratar, de modo distinto, las posibilidades y características de cada proyecto de emprendimiento, enfocándose en aquellos con mejor posibilidad de crear valor económico y social, con la finalidad de generar mayor impacto en las condiciones de vulneración de derechos y pobreza del país. Esta invitación a la diferenciación, que hace Piedrahita (2019), conduce a pensar en la importancia de reconocer las diferencias en las poblaciones con las cuales se realiza intervención social, a través de proyectos de emprendimiento; y los resultados de esta investigación ponen sobre la mesa la importancia de reconocer "lo diferente", que remite a no asumir que existe un modo único de emprender que aplicaría para todos.

Teniendo en cuenta lo anterior, los participantes de esta investigación permitieron develar que, en los procesos de emprendimiento, se requiere dar lugar a la subjetividad, a lo singular de cada persona y eso que aparece en ocasiones como falla, como síntoma. En los programas de intervención se denomina "deserción" o "el porcentaje de beneficiarios que no cumplió los indicadores". Esto puede ser susceptible de ser abordado desde una perspectiva psicosocial y de las trayectorias diversas del sujeto emprendedor que "siga las coordenadas dadas por el saber de cada sujeto, uno por uno, tomando distancia de los procesos protocolizados y masificados" (Castro-Sardi y Munévar, 2018, p. 106).

A partir de los resultados de esta investigación-intervención, se propone una perspectiva que incluya la historia y experiencia subjetiva en los procesos de capacitación para el emprendimiento. Una mirada que tenga en cuenta la diversidad y pluralidad de sentidos que tiene el emprendimiento en la vida de una persona; que no deje de lado la experiencia y la historia singular de todo sujeto emprendedor o emprendedora. Una mirada que pueda introducir un no-todos emprendedores o, al menos, no-todos del mismo modo.

\section{Referencias}

Álvarez, C., Noguera, M. y Urbano, D. (2012). Condicionantes del entorno y emprendimiento femenino. Un estudio cuantitativo en España. Revista Economía Industrial, (383), 43-52.

Ángel, D. A. (2011). La hermenéutica y los métodos de investigación en ciencias sociales. Estudios Filosóficos, 44(5), 9-37.

Annan, J., Blattman, C., Green, E. y Jamison, J. (2015). Women's entrepreneurship and intimate partner violence: A cluster randomized trial of microenterprise assistance and partner participation in post-conflict Uganda. Social Science \& Medicine, (133), 177-188. http://dx.doi.org/10.1016/j. socscimed.2015.03.042

Arbeláez, N. (2019). El emprendimiento, como se maneja hoy, contribuye a tener personas más pobres. La Silla Académica. https://lasillavacia.com/silla-academica/universidad-externado-colombia/ emprendimiento-como-se-maneja-hoy-contribuye-tener 
Bauman, Z. (2005). Vidas Desperdiciadas: La Modernidad y sus Parias. Paidós Ibérica.

Bueno-Restrepo, J. M. (2012). Ecos del deseo en el cuerpo, el enigma de la autoinmunidad. Affectio Societatis, 9(17), 2-13.

CAPsi-Icesi -Consultorio de Atención Psicosocial, Universidad Icesi-. (2018). Informe final. Estrategia de atención psicosocial en salud mental. Documento no publicado.

Carter, S. (2000). Improving the numbers and performance of women-owned business. Some implications for training and advisory services. Education and training, 42(4-5), 326-334.

Castillo, M. I. y Gómez, E. (2004). Las peculiaridades de la investigación en Psicoanálisis. Terapia Psicológica, 22(1), 25-32.

Castro-Sardi, X. (2013). Salud mental sin sujeto. Sobre la expulsión de la subjetividad de las prácticas actuales en salud mental. CS, (11), 73-114. https://doi.org/10.18046/recs.ill.1567

Castro-Sardi, X. (2014) El sujeto del psicoanálisis. Más allá de la dicotomía individuo-sociedad. Affectio Societatis, 11(21), 102-121.

Castro-Sardi, X. (2016). Salud mental y atención psicosocial. Reflexiones a partir de la experiencia de un dispositivo de escucha y su impacto en la rehabilitación de la cronicidad mental. En O. A. Bravo (Ed.), Pensar la salud mental: aspectos clínicos, epistemológicos, culturales y políticos (pp. 122-157). Universidad Icesi.

Castro-Sardi, X. y Munévar, M. (2016). Consultorio de Atención Psicosocial: clínica y lazo social. En Colegio Colombiano de Psicólogos (Ed.), Experiencias Significativas en Salud Mental y Psicología (pp. 16-29). Colegio Colombiano de Psicólogos. http://colpsic.org.co/aym_image/files/ EXPERIENCIAS_SIGNIFICATIVAS.pdf

Castro-Sardi, X. y Munévar, M. (2018). Escuchando a las víctimas del conflicto armado colombiano: la experiencia de un dispositivo de atención psicosocial. CS, (25), 81-109. http://dx.doi.org/10.18046/ recs.i25.2220

Clark, L., Labuzetta, J. N., Lawrence A., Sahakian, B. y Vyakarnum, S. (2008). The innovative brain. International Journal of Science: Nature, 456(7219), 168-169. https://doi.org/10.1038/456168a

Congreso de Colombia. (2013, 21 de enero). Ley 1616 de 2013. Ley de Salud Mental. DO: 48.680

Dahl, M. S., Nielsen, J. y Mojtabai, R. (2010). The effects of becoming an entrepreneur on the use of psychotropics among entrepreneurs and their spouses. Scandinavian Journal of Public Health, 38(8), 857-63. https://doi.org/10.1177/1403494810375490

De Castro-Korgi, S. (2011). Del duelo al deseo. Revista Desde el jardín de Freud, (11), 95-114.

Desviat, M. (2015). De la reforma psiquiátrica a la salud mental colectiva. Grupo 5.

Díaz, D., Rodríguez-Carvajal, R., Blanco, A., Moreno-Jiménez, B., Gallardo, I., Valle, C. y Dierendonck, D. (2006). Adaptación española de las escalas de bienestar psicológico de Ryff. Revista Psicothema, 18(17), 572-577.

Dolan, C. y Rajak, D. (2016). Remaking Africa's Informal Economies: Youth, Entrepreneurship and the Promise of Inclusion at the Bottom of the Pyramid. Journal of Development Studies, 52(4), 514-529. https://doi.org/10.1080/00220388.2015.1126249 
¡Todos(as) emprendedores(as)! Subjetividad y salud mental en un proceso de capacitación en emprendimiento

Eslava, M. (2019). Colombia no necesita más emprendimiento, sino más impacto. La Silla Vacía. https://lasillavacia.com/silla-llena/blogoeconomia/ colombia-no-necesita-mas-emprendimiento-sino-mas-impacto-70623

Fowle, M. (2019). The Entrepreneurial Dream: Happiness, Depression and Freedom. International Review of Entrepreneurship, 17(3), 305-322.

Freud, S. (2004). Más allá del principio del placer (1920). En Obras completas volumen XVIII (pp. 1-62). Amorrortu Editores

Galeano, E. (2004). Estrategias de investigación social cualitativa: El giro de la mirada. La Carreta Editores.

Galende, E. (1997). Un horizonte incierto, psicoanálisis y salud mental en la sociedad actual. Paidós.

Galende, E. (2008). Psicofármacos y salud mental: la ilusión de no ser. Lugar Editorial.

Galende, E. (2015). Conocimiento y prácticas en Salud Mental. Lugar Editorial.

Gallo, H. (2011). Feminidades: sacrificio y negociación en el tiempo de los derechos. En D. Carmona (Comp.), El Asunto del Método en la Investigación Psicoanalítica (pp. 99-125). Editorial Universidad de Antioquía.

GERA -Global Entrepreneurship Research Association-. (2018). Global Entrepreneurship Monitor: Global Report 2017/18. GERA.

GERA -Global Entrepreneurship Research Association-. (s.f). How GEM Defines Entrepreneurship. GERA.

González-Rey, F (2006). Investigación Cualitativa y Subjetividad. ODHAG.

Gunnarsson, K., Josephson M. y Vingård, E. (2007). Self-rated health and working conditions of small-scale enterprises in Sweden. Industrial Health, 45(6), 775-780. https://doi.org/10.2486/ indhealth.45.775

Holguín, C. M. (2012). Malestares en la cultura: ¡Tú debes ser feliz! Radar nº 95. http://www.nelmexico.org/articulos/seccion/radar/edicion/100/606/Malestares-en-la-cultura-Tu-debes-ser-feliz-

Jaramillo, A. M. (2013). Las Mujeres: entre el sacrificio y la negociación. Boletín de Antropología, 28(45), 62-75.

Johansson, S. y Sjindjapkin, A. (2015). The Socially Empowering Impact of Entrepreneurship: A Study on Urban Ugandan Women. Linnaeus University.

Kabeer, N. (1999). Resources, Agency, Achievements: Reflections on the Measurement of Women's Empowerment. Development and Change, 30(3), 435-464. https://doi.org/10.1111/1467-7660.00125

Lederman, D., Messina, J., Pienknagura, S. y Rigoloni, J. (2014). Resumen del libro del Banco Mundial titulado El emprendimiento en América Latina: muchas empresas y poca innovación. Banco Internacional de Reconstrucción y Fomento; Banco Mundial. https://www.worldbank.org/ content/dam/Worldbank/document/LAC/EmprendimientoAmericaLatina_resumen.pdf

Louie, R. K. (2015). The Psychiatry of Entrepreneurship. Academic Psychiatry, 40(2), 386-388. https:// doi.org/10.1007/s40596-015-0288-y 
Marulanda, F. y Morales, S. (2016). Entorno y motivaciones para emprender. Revista Escuela de Administración de Negocios, (81), 12-28. http://dx.doi.org/10.21158/01208160.n81.2016.1556

Miller, J. A. (2003). Introducción al Método Psicoanalítico. Editorial Paidós.

Moreno, M. A. (2013). Psicoanálisis e intervención social. CS, (11), 115-142. https://doi.org/10.18046/recs. ill.1568

Narayan, D. (2005). Conceptual Framework and Methodological. En D. Narayan (Ed.), Challenges Measuring Empowerment: cross-disciplinary Perspectives (pp. 3-38). The International Bank for Reconstruction and Development.

North, D. C. (2005) Understanding the process of economic change. Princeton University Press.

Peláez-Jaramillo, G. P. (2011). El sujeto y el lazo social en el psicoanálisis. Affectio Societatis, 8(15), 1-9.

Piedrahita, E. (2019). Ni es lo mismo, ni es igual. Revista Semana. https://www.semana.com/opinion/ articulo/ni-es-lo-mismo-ni-es-igual--columna-de-esteban-piedrahita/607896

Rowlands, J. (1997). Questioning Empowerment: Working with Women in Honduras. Oxfam.

Sorenson, R. L., Folker, C. y Brigham, K. H. (2008). The Collaborative Network Orientation: Achieving Business Success Through Collaborative Relationship. Entrepreneurship Theory and Practice, 32(4), 615-634. http://dx.doi.org/10.1111/j.1540-6520.2008.00245.x

Stephan, U. (2018). Entrepreneurs' Mental Health and Well-Being: A Review and Research Agenda. Academy of Management Perspectives, 23(3), 2-69. https://doi.org/10.5465/amp.2017.0001 